\title{
Experimental Formation of Gallstones by Means of Roundworm Eggs
}

\author{
By \\ Tokuo Atsumi \\ (熱海 徳 夫) \\ From the Surgical Clinic of Prof. T. Maki, School of Medicine, \\ Hirosaki University, Hirosaki
}

(Received for publication October 29, 1955)

Invasion of roundworms into the biliary tract is a routine matter of observation ${ }^{1)}$ in Japan, and it is well known that the cuticles of such invading roundworms become nuclei for the formation of gallstones, on which Sato $^{21}$ of our clinic has already published his report. The eggs left in the biliary tract by such worms also play a part in stone formation. Yoshida ${ }^{3)}$ for the first time reported that some gallstones he had observed microscopically were formed, making ascaris eggs their nuclei, in 1908. Yasuda ${ }^{4}$ of our clinic chemically dissolved the nuclear parts of apparently ordinary pigment chalk stones and detected many ascaris eggs in a very high frequency. Figures of stone formation in the initial stage with pigment chalk precipitation on the shells of ascaris eggs were clinically obtained, by Maruyama ${ }^{5)}$ and Asakura $^{6)}$ of our clinic. This series of findings suggests that the ascaris eggs show a deep significance as nuclei in pigment chalk stone formation in Japan. On the other hand, Asakura ${ }^{7}$ has accomplished a crystallographic study of the ascaris egg shells and has published a very suggestive observation that the shells of fertilized ascaris eggs are, of all eggs of human parasites, the best adapted for forming nuclei of gallstones. In good agreement with his results, the author of this paper has succeeded in experimentally precipitating pigment chalk on ascaris eggs and further observing the mode and process of the precipitation, as reported in the following.

\section{EXPERIMENTAL}

\section{Method}

1) Ascaris eggs used: These were directly extracted from the uteri of female ascaris. The extracted mass contained fertilized and unfertilized eggs with and without albuminoid membrane and these were used in suspension in physiological saline. 
2) Animals used: 38 male and 31 female mongrel Japanese dogs in normal health and 3-11 kg. in body weight were used. No special manipulation or restriction was given their feed, either before or after the operation, but the usual table leavings were fed throughout.

3) Grouping: In all the tests, $0.5-1.0 \mathrm{cc}$ per $\mathrm{kg}$ body weight of $4 \%$ morphine hydrochloride solution was used for basal narcosis in combination with local anesthesia with $0.5 \%$ procaine hydrochloride. The animals were divided into the three following groups according to the experimental methods applied.

Group I. Ascaris eggs directly introduced into the gallbladder.

39 dogs were subjected to this method. Upon laparotomy, the gallbladder was punctured and the cystic duct was lightly ligated with silken threads, so that stasis was occasioned in the bladder without complete closure of the duct. Then, the ascaris egg suspension was introduced with a syringe directly into the gallbladder. 25 of the subjects had only ascaris eggs thus introduced, but the other 14 had $1.0 \mathrm{cc}$ of calcium chloride injected in combination with the egg suspension.

Group II. Ascaris eggs introduced into the choledochus per duodenum. 30 dogs were used here. The egg suspension was introduced through a Nelaton's catheter No. 3 temporarily inserted transduodenally into the Vater's papilla. In this group, the cystic duct was left unligated in 15 dogs, half ligated, as in the above group, in 7 and in 8 cases it was completely ligated or cholecystectomy performed, for the purpose of observing the ascaris eggs in the biliary tract only in exclusion of the influence of the gallbladder.

Group III. Controls. With these the three following experiments were carried out: i. Foreign objects other than ascaris eggs were introduced into the gallbladder. These objects were sterilized objects, such as powdered charcoal, white sand grains, shreds of rubber tissue, each being about $1-3 \mathrm{~mm}^{3}$ in size and $1.0 \mathrm{~g}$. in total quantity. Each object was directly introduced into the gallbladder through an incision and the cystic duct was lightly ligated as in Group I above (10 dogs). ii. The ascaris egg suspension was merely introduced directly into the gallbladder, but no manipulation was applied to the cystic duct and the Vater's papilla (9 dogs). iii. In vitro: Samples of human gallbladder bile extracted at the time of surgical operations of the liver or the biliary tract diseases were centrifuged and sedimentated, and after attesting their freedom from eggs and cuticles of parasites, were mixed with ascaris egg suspension and kept in an incubator at $37^{\circ}$, for microscopic examinations at stated periods.

In all the above experiments, the bladdar bile was sampled before the experimental operations to assure that it was free from eggs of parasites and bile sand.

4) Appraisal of the results: After the lapse of the stated time, 
the animals were operated upon for the second time, their bile in the gallbladder or the choledochus being extracted, and then the ascaris eggs in it were examined. In the case when pigment chalk, which seemed precipitation was found on them, the color, the form and the size of the granules were observed, their bilirubin reaction examined under a microscope under low magnification, using Kossa's method in the examination of the precipitate. In the fields where positive ascaris eggs were found, the frequency of the eggs with and without the precipitation was determined as classified by the sorts of the eggs. The properties of the bile, namely its $\mathrm{pH}$ value and its bacterial count were also examined at the same time.

\section{Results}

\section{Process of pigment chalk (calcium bilirubinate) precipitation on ascaris eggs}

Of all the 69 tested subjects, precipitation of pigment chalk granules on different kinds of ascaris eggs was observed in 14 cases or $20 \%$ in total. Under a microscope, these are observed as granules of dark brown color $3-5 \mu$ in diameter-showing figures entirely identical with the pigment chalk granules found precipitated on ascaris eggs and cuticles in clinical cases. A test of these granules for bilirubin under the above mentioned method showed that they were rich in bilirubin. The precipitation showed some difference according to the sort of the eggs.

\section{Fertilized eggs with albuminoid membrane}

Precipitation was most conspicuous on these. In the early stage, the a'buminoid membrane, the egg shells, and the egg cells were clearly observable, granules of calcium bilirubinate being sporadically precipitated on them. Fige. 1 and 2 show such eggs 4 days after their introduction into the gallbladder. In some cases, the precipitation become aggregated and dense as early as 7 days after the implantation, so that the egg shell becomes hidden. In about 3 weeks, the precipitation progresses further and the egg shells and the egg cells become indistinct (Fig. 3, 4). One month after the introduction of the eggs, the precipitated pigment chalk covers up the eggs in one aggregated mass and shows a figure of incipient gallstone (Fig. 5).

II. Fertilized eggs without albuminoid membrane

Precipitation of calcium bilirubinate was observed first on the 7th day (Fig. 6), and Fig. 7 shows such an egg on the 18th day. No such advanced precipitation as in the above was observed with these eggs.

III. Precipitation of calcium bilirubinate on egg cells

In fertilized but albuminoid membraneless eggs, it was often seen that the bile first penetrates into the egg cell and then the calcium bilirubinate precipi- 
tation apparently has grown to cover the egg shell (Fig. 8).

Fig. 1

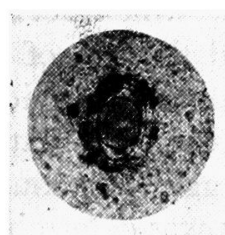

Fig. 2

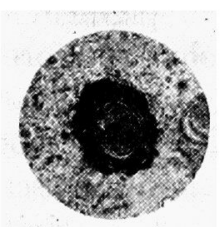

Fig. 3

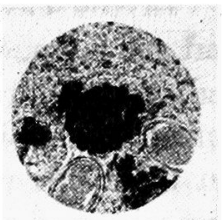

Fig. 4

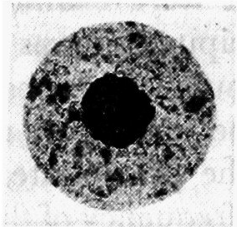

Fig. 1. The initial stage of pigment chalk precipitation on egg shell, 4 days after the insertion of fertilized ascaris eggs with albuminoid membrane into the gall bladder.

Fig. 2. The same as the above.

Fig. 3. Pigment chalk precipitation in the next stage, 18 days after the insertion.

Fig. 4. Another instance of the same, 18 days after insertion.

Fig. 5

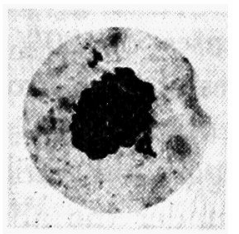

Fig. 6

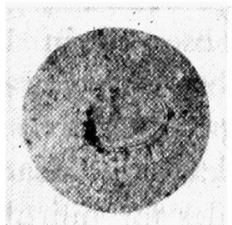

Fig. 7

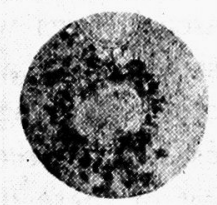

Fig. 8

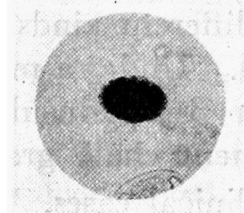

Fig. 5. One of the eggs, one month after insertion.

Fig. 6. One instance of precipitation, 7 days after the insertion of fertilized ascaris eggs without albuminoid membrane into the gall bladder.

Fig. 7. The same, showing aggregation of pigment chalk granules around the egg. A picture taken 10 days after the insertion.

Fig. 8. Pigment chalk precipitation in egg cells, 8 days after the insertion of fertilized membraneless eggs into the gall bladder.

\section{Unfertilized eggs}

Only in one instance of unfertilized eggs with albuminoid membrane was observed a slight precipitation. No precipitation was ever observed on unfertilized eggs without albuminoid membrane.

\section{Results by groups}

I. Group I : Precipitation was observed in 7 or $17.9 \%$ of the 39 animals of this group. Of the 25 animals inoculated only with ascaris egg suspension, 2 cases, and of the 14 animals inoculated with calcium chloride solution in combination with the suspension, 5 cases showed precipitation of calcium bilirubinate on the eggs.

II. Group II : Precipitation was observed in 7 or $23.3 \%$ of the 30 animals. Positive results were obtained in 3 of the 15 dogs with their cystic ducts left unligated and in 3 of the 7 dogs with their cystic ducts lightly ligated. 
In 1 case among these positive cases, the precipitation was found on ascaris eggs in the choledochus. In the subgroups where the gallbladder was extirpated or the cystic duct was completely closed, precipitation was observed on one case only on eggs in the choledochus.

III. Control experiments: a. When foreign objects other than ascaris eggs were used, no accretion of the foreign matter or precipitation of calcium bilirubinate on them was observed.

b. When merely ascaris eggs were introduced directly into the gallbladder, without giving any manipulation to the cystic duct or the region of the Vater's papilla, no precipitation of calcium bilirubinate was ever observed either on eggs in the biliary ducts or on those in the gallbladder.

c. In vitro experiments, precipitation was observed in 4 cases among 10. The bile used in these 4 positive tests was taken from 4 cases, one from cases of ascariasis of biliary tract, and another from liver abscess cases, and the others from cases of cholecystitis. All the eggs on which such a precipitation was observed were fertilized eggs. The earliest precipitation in vitro occurred one month after the addition of bile.

\section{Relation between sorts of ascaris eggs and frequency of precipitation}

In all the above experiments, fertilized eggs with albuminoid membrane showed the highest percentage of precipitation $(68.5 \%)$, followed in percentage by fertilized albuminoid membraneless eggs $(29.9 \%)$ and unfertilized eggs with albuminoid membrane $(1.5 \%)$, no instance of precipitation on unfertilized membraneless eggs being observed.

\section{Relation between precipitation of calcium bilirubinate and bile stasis}

There was no marked difference in the frequency of precipitation observed in Groups I and II, but in the Group II, the frequency was larger in the animals with their cyctic ducts lightly ligated (3 positive cases among 7) than in those with their cystic ducts left intact ( 3 positive cases among 15), while with the animals not operated upon to cause stasis of their bile in the Group III, no instance of precipitation was ever observed.

\section{Properties of bile}

I. $\mathrm{pH}$ values: Of the 69 animals in the Groups I and II above, the $\mathrm{pH}$ values of their bile prior to and following the operation were determined with 44 cases. In the majority of the cases (33 among 44), the $\mathrm{pH}$ values of their bile showed a tendency to acidification. The details by groups and occurrence of calcium bilirubinate precipitation were as follows: 
a. In Group $\mathrm{I}$, the $\mathrm{pH}$ values tended to acidity in 5 of the 7 cases that showed precipitation and in 12 of the 19 cases that showed negative results whose change in $\mathrm{pH}$ values of bile was clearly observed.

b. In Group II, all the cases of which the change in $\mathrm{pH}$ values were followed up showed a turn to fall, except two cases, one from among the 6 cases with positive and the other from among 12 cases with negative results in the precipitation tests.

c. The change in $\mathrm{pH}$ was recorded with 8 cases of foreign matter inclusion tests in the control experiments, of which 6 cases showed acidification of the bile.

II. Bacteria in the bile : In Groups I and II, the bacterial counts were determined by cultures using bile from the gallbladder and the biliary ducts taken before and after the operation performed on 53 cases, including the cases in Group II where the ascaris eggs in the biliary ducts were examined, of which the counts were taken only of the duct bile. The detected bacilli chiefly consisted of colibacillus, no other kinds of bacilli being observed except some staphylococci albi found in a few cases only. Among the 14 cases where calcium bilirubinate precipitation was demonstrated, bacteria in duct bile were detected in 11 cases, of which colibacilli were detected in 4 of the 5 tested cases in Group $I$ and in 5 of the 6 tested cases in Group II. Staphylococci albi were detected only in one case of the dogs inoculated with ascaris egg suspension only in Group I, and in this case also colibacilli were found in mixed infection. No marked difference was found in the frequency of detection of colibacillus between the cases with positive $(81.8 \%)$ and negative $(75.5 \%)$ calcium bilirubinate precipitation.

\section{Summary And Discussion}

As detailed in the above, the author made the experiment of introducing ascaris eggs into the gallbladder or into the biliary ducts of dogs and causing stasis of bile in them, and detected precipitation of calcium bilirubinate in $20 \%$ of the total tested cases. It is generally considered ${ }^{8)}$ that in $\operatorname{dog}$ the experimental formation of gallstones is very difficult, so the above experiment showing a high percentage of successful cases is worthy of our attention.

As reliable reports demonstrating ascaris eggs detected in gallstones, we can point to those by Yamamoto, ${ }^{9)}$ Nomamoto $^{10)}$ and Nishimura, ${ }^{11}$ besides that by Yoshida ${ }^{3)}$ cited above, but as to experimental studies on the relation between eggs of parasites and gallstone formation, we can count only those reported by Inoue ${ }^{12)}$ and $\mathrm{Kono}^{13)}$ who have taken the part played by the eggs of clonorchis sinensis in gallstone formation in the liver as the postulate of their studies. Hepatic clonorchiasis is rather 
frequent in Japan, but the part played by ascaris lumbricoides is far more important in respect to surgical diseases of the biliary tract. In the above experiment I could show that the eggs of ascaris lumbricoides like its cuticles have a close connection with the formation of pigment chalk stones which is characteristic of gallstone in Japan.

It must be noted that precipitation of calcium bilirubinate is more apt to be incurred by fertilized eggs of ascaris. This is, in the first place, due to the higher crystallographic stability of the structure of the shell of a fertilized egg than that of an unfertilized egg, and the consequent better adaptation of the former as a nucleus of a gallstone. In the second place, the albuminoid membrane also has, as Asakura states, a great significance for the precipitation of calcium compound, when it putrefies and calcium carbonate deposit takes place on it. The utterly negative results of the experiments with foreign objects other than ascaris eggs may be taken to endorse the high suitableness of ascaris eggs as nuclei of pigment chalk stones. The control experiments with ascaris eggs introduced in the gallbladder, no stasis of bile being induced in it, produced scarcely any case showing stony precipitation, indicating the strong influence of bile stasis on stone formation. Fukushima ${ }^{14}$ of our clinic has proved experimentally that an invasion of an ascaris into the common bile duct causes a strong contracture of the Oddi's muscle by mechanically stimulating the Vater's papilla with the accompanying bile stasis and dilatation of the biliary ducts. This fact is in agreement with the rather frequent occurrence of precipitation in Group II than in Group I above.

It must be also considered that in these experiments, the dogs used were fed with the usual left-over rice and no manipulation or restriction was placed on their feed. In the past, we have heard discussions on the influence of diet on gallstone formation, but the above experiments show that even if the influence of the general bodily conditions does not come into consideration, if only the local requirements are fulfilled, precipitation of calcium bilirubinate can take place. This precipitation may take place as much in the gallbladder as in the biliary ducts, the only difference lying in the time when the precipitation begins-4-5 days in the former and 7-10 days in the latter at the earliest, after the entrance of the ascaris eggs.

Taking the above experimental results into consideration, we may conclude that, in ascariasis of the biliary tract, when ascaris eggs are laid in the tract, a foundation for a very likely and prompt formation of gallstones is laid. We may also find some explanation in this for the frequency of gallstones being found in the biliary ducts and the liver in Japan.

Next, I will speak a word on the properties of the bile.

According to $\mathrm{Oba},{ }^{15)}$ the $\mathrm{pH}$ value of normal dogs ranges between 
6.48 and 7.52. In the above experiments, the value stood between 6.41 and 7.34, but after the operation for the introduction of ascaris eggs, it tended to decrease to the acid side. It has been pointed out by many researchers that a tendency to acidification of bile causes precipitation or coagulating out of its components and thus becomes an important factor in gallstone formation. In the above experiments, the acidification of the bile must have also played an outstanding part in gallstone formation.

The rate of bacilli detection in the bile of cholelithiasis cases is generally higher in Japan than in Western countries, colibacillus occupying the leading position among the detected bacilli, presumably by ascendant infection. In the above experiments, there was no perceptible difference in the frequency of bacilli detection between the bile in which precipitation of calcium bilirubinate on ascaris eggs was observed and that wherein no precipitation was found $(81.8 \%: 75.7 \%)$. This suggests that the colibacillus infection, rather than playing a directly important part in stone formation, exerts a secondary influence on the composition of the bile through inducing inflammatory changes in the adjacent tissues, and this influence results in materializing conditions favorable for stone formation. As bilirubin must combine with calcium before precipitating, much calcium is required for pigment chalk stone formation. In Group I above precipitation was slightly more frequent in the animals injected with calcium chloride solution in combination with ascaris egg suspension than in the other animals. Now, it has been demonstrated by Hashimoto ${ }^{16)}$ of our clinic that the calcium content in the bile is increased in the initial stage of inflammation or stasis of the gallbladder.

\section{ConCLUSION}

As a link in the series of experimental studies concerning gallstone formation due to roundworm, the following experiments were conducted with dogs as subjects. Ascaris eggs were introduced into the gallbladder and the biliary ducts of dogs, the consequent stasis induced in them by the application of various methods, and then the eggs were taken out by reoperation at stated intervals. Finally the eggs and the composition of bile were examined. The results obtained were as follows:

1. Though no manipulation or restriction was applied to the feed of the dogs, precipitation of calcium bilirubinate on the ascaris eggs was observed on $20 \%$ of the total cases thus examined.

2 . In most of the experimented animals, infection by colibacillus was observed in the biliary tract, but the most important factor causing the precipitation of calcium bilirubinate in these experiments was presumable the stasis of bile. 
3. Of the different conditions of the eggs, fertilized eggs were more frequently subject to precipitation of calcium bilurubinate than unfertilized eggs, and among the former, those with albuminoid membranes were most apt to receive precipitation on them.

4. Of the different groups of the experimental animals, the group injected with calcium chloride solution in combination with the ascaris egg suspension and with their cystic ducts lightly ligated showed the highest frequency of precipitation.

5. In general, in the early stage following the introduction of ascaris eggs, calcium bilirubinate is found to have precipitated in sporadic dots on the albuminoid membranes and the shells of the eggs, so that these and the egg cells are still clearly visible, but in 3 weeks, the precipitation will gain in scope and density, so that the structure of the eggs will be blurred. The time required for the initiation of the precipitation after the introduction of the eggs was 4-5 days in the gallbladder and 7-10 days in the biliary ducts, while it took 30 days for the first precipitation to occur in vitro.

6. In some cases of fertilized but albuminoid membraneless eggs, calcium bilirubinate seemed first to seep into the egg cell before precipitation on the egg shell, and to grow thence toward the shell.

7. From the above results of the experiments, I am led to the conclusion that, in ascariasis of the biliary tract, when ascaris eggs are laid or left in the biliary tract, a groundwork has already been laid for a rapid formation of pigment chalk stones in a very high probability.

Thanks are due to the Education Ministry for the grant from the Scientific Research Fund to this study. T. Maki.

\section{References}

1) Maki, Tokyo Iji Shinshi (Jap.), 1952, 69, 6.

2) Sato, Hirosaki Igaku Zasshi (Jap.), 1955, 6, 194.

3) Yoshida, Tokyo Iji Shinshi (Jap.), 1908, 1551, 365.

4) Yasuda, Hirosaki Igaku Zasshi (Jap.), 1955, 6, 391.

5) Maruyama, ibid., 1954, 5, 97.

6) Asakura, ibid., 1954, 5, 102.

7) Asakura, Tohoku Jour. exp. Med., 1956, 64, 105, 117.

8) Miyake, Saishin Igaku (Jap.), 1948, 3, 456.

9) Yamamoto \& Sato, Nippon Geka Gakkai Zasshi (Jap.), 1917, 18, 192.

10) Nomamoto, Igaku Kenkyu (Jap.), 1944, 18, 415.

11) Nishimura, Rinsho Geka (Jap.), 1948, 3, 145.

12) Inoue \& Ikeda, Jikken Shokaki Byogaku (Jap.), 1938, 13, 814.

13) Kono, ibid., 1942, 17, 420.

14) Fukushima, Hirosaki Igaku Zasshi (Jap.), 1955, 6, 58. 
15) Oba, Okayama Igakkai Zasshi (Jap.), 1933, 45, 1.

16) Hashimoto, Hirosaki Igaku Zasshi (Jap.) 1956, 7, 323. 